\title{
Limits to the presence of transiting circumbinary planets in CoRoT Data ${ }^{\star}$
}

\author{
P. Klagyivik ${ }^{1,2,3}$, H. J. Deeg ${ }^{1,2}$, J. Cabrera ${ }^{4}$, Sz. Csizmadia ${ }^{4}$, and J. M. Almenara ${ }^{5}$ \\ 1 Instituto de Astrofísica de Canarias, C. Vía Láctea S/N, 38205 La Laguna, Tenerife, Spain \\ e-mail: pklagyi@gmail.com; hdeeg@iac.es \\ 2 Universidad de La Laguna, Dept. de Astrofísica, 38206 La Laguna, Tenerife, Spain \\ 3 Konkoly Observatory, Research Centre for Astronomy and Earth Sciences, Hungarian Academy of Sciences, \\ Konkoly Thege Miklós út 15-17, 1121 Budapest, Hungary \\ 4 Institute of Planetary Research, German Aerospace Center, Rutherfordstrasse 2, 12489 Berlin, Germany \\ 5 Observatoire Astronomique de l'Université de Genève, 51 chemin des Maillettes, 1290 Versoix, Switzerland
}

Received 3 February 2016 / Accepted 25 October 2016

\begin{abstract}
Aims. During its flight phase, from 2007-2012, the CoRoT mission delivered light curves for over 2000 eclipsing binaries. Data from the Kepler mission have proven the existence of several transiting circumbinary planets. While light curves from CoRoT typically have lower precision and shorter coverage, the number of CoRoT targets is similar to that of Kepler and some of the known circumbinary planets could potentially be detected in CoRoT data as well. The aim of this work was to reanalyse the entire CoRoT Data set to search for the presence of circumbinary planets and to derive limits on the abundances of such planets.

Methods. We developed a code that removes the signatures of eclipsing binaries from the light curves, and searches for quasi-periodic, transit-like features in the light curves after removal of binary eclipses and instrumental features. The code requires little information on sample systems and can also be used for other space missions, such as Kepler, K2, TESS, and PLATO. The code is broad in the requirements leading to detections, but was tuned to deliver an amount of detections that are manageable in a subsequent, mainly visual, assessment of their origin.

Results. We identified three planet candidates in the CoRoT sample whose transits would have arisen from a single pass across the central binary; however, no candidates with transit events from multiple planetary orbits remained. We calculated the upper limits for the number of Jupiter, Saturn-, and Neptune-sized planets in co-planar orbits for different orbital period ranges. We found that there are much fewer giant planets in short periodic orbits around close binary systems than around single stars.
\end{abstract}

Key words. planets and satellites: detection - techniques: photometric - binaries: eclipsing

\section{Introduction}

Several years before the detection of the first extrasolar planet, eclipsing binary systems (EBs) were already suggested as prime targets for the detection of transiting planets (Borucki \& Summers 1984; Schneider \& Chevreton 1990; Schneider 1994; Hale \& Doyle 1994). This predilection for EBs came from the assumption that circumbinary planets (CBPs), that is, planets orbiting around both binary components, would have orbital planes that are likely aligned with the binary plane. Because EBs are defined as binaries for which we can observe eclipses, this means that their planets would have a high chance of causing observable eclipses or transits - an argument that has recently been reinforced by an analysis of CBP transit probabilities by Martin \& Triaud (2015).

Early on, the eclipsing M-star binary CM Draconis was identified as the best object for a targeted transit search (Schneider \& Doyle 1995), which was initiated as the TEP (Transits of Extrasolar Planets) project during the years 19942000 (Doyle et al. 1996, 2000; Jenkins et al. 1996; Deeg et al. 1998). The TEP project remained the only search for transiting

* Full Table 1 is only available at the CDS via anonymous ftp to cdsarc.u-strasbg.fr (130.79.128.5) or via

http://cdsarc.u-strasbg.fr/viz-bin/qcat?J/A+A/602/A117
CBPs until the arrival of CoRoT, when an early part of CoRoT Data was analysed for the presence of CBPs by Almenara (2010). While none of these searches found any CBPs, shortly after the detection of Kepler-16b (Doyle et al. 2011) proved both the existence of CBPs and the validity of transits as a method to detect them. Further detections - all of them arising from $\mathrm{Ke}$ pler mission data - have presently led to a total of ten transiting $\mathrm{CBPs}^{1}$. Including recent discoveries of CBPs by other methods, notably imaging (e.g. Kuzuhara et al. 2011) and eclipse timing (e.g. Beuermann et al. 2010; Potter et al. 2011; Baran et al. 2015), a total of 25 CBPs are currently known and CBPs have become a very active sub-discipline of exoplanet research.

The inner edge of a stable planetary orbit around a binary system has an orbital period of $\sim 3$ times the orbital period of the binary (Dvorak et al. 1989; Holman \& Wiegert 1999). Therefore, CBPs close to the inner stability limit in short periodic binary systems should be more readily detectable than those around longer period binaries. This is brought about by the higher probability of CBPs closer to the central binary to have an inclination suitable for observable transits, as well as by the higher frequency of transits from CBPs on shorter-periodic orbits. These detection biases are similar to planets transiting

\footnotetext{
1 CBP counts are based on entries in the NASA Exoplanet Archive
} with a circumbinary flag of 1 , as of 20 January 2017. 
single stars, where short periodic planets are more readily detectable. However, in a CBP search, there is also a bias against short-periodic planets, since short-periodic EBs will be tidally locked, which implies fast stellar rotation rates that induce larger photometric activity.

None of the CBPs detected by Kepler had central binaries with periods $<7$ days. This lack of planets orbiting short period EBs is apparently real and not due to difficulties in their detection. Martin et al. (2015) and Hamers et al. (2016) argue that short periodic binaries form in triple systems and are followed by a dynamical evolution that either ejects their planets, or moves them to wide and potentially inclined orbits. An additional search on a large sample for the presence of shorter periodic CBPs, which can only have stable orbits around correspondingly shorter periodic binaries, is therefore of interest as it may support the hypothesis that short periodic CBPs are absent or very rare.

The CoRoT space telescope during its nearly six years of activity observed approximately the same number of targets as Kepler; the number of surveyed EBs are also similar. However, there are some differences that reduce the chances of finding transits in the CoRoT eclipsing binary light curves. The photometric noises of CoRoT are $\approx 4 \times$ larger than those of $\mathrm{Ke}$ pler for a given brightness (Aigrain et al. 2009, for CoRoT and Gilliland et al. 2010; Jenkins et al. 2010, for Kepler), and the time coverage of observations by CoRoT is between 30 and 180 days, depending on the observational run, in contrast to the $\approx 4 \mathrm{yr}$ of Kepler. Detection probabilities in the CoRoT sample are therefore more heavily tilted towards the discovery of shorter periodic planets than data from Kepler. This can be noted in their discoveries of planets around single stars, where the longest periodic CoRoT planet is CoRoT-9b with $p=95$ days (Deeg et al. 2010), whereas the longest periodic Kepler planet is Kepler$455 \mathrm{~b}$ with $p=1322$ days (Wang et al. 2015a, there given as KIC 3558849 b).

The first search for CBPs in CoRoT Data by Almenara (2010) was limited to the data that were available in 2008, namely the CoRoT initial run (IRa01) and the first two long runs (LRc01 and LRa01). This search was performed with a matched filter detection algorithm based on that of Doyle et al. (2000) used in the TEP project, which had been developed for a deep search of a single, well-characterised target. The algorithm intended to model the CBP transit signatures in a detailed way, while it stepped through the orbital periods and phases of the potential planets. The algorithm required therefore rather good knowledge of the binaries that were analysed, in particular of their masses and sizes. Consequently, only a set of ten binaries from CoRoT Data were searched in detail. These were selected by requiring detached and deep eclipses, a high $\mathrm{S} / \mathrm{N}$, and a relatively short orbital period to maintain $P_{\text {min }}<T_{\text {cov }}$, where $P_{\text {min }}$ is the period of the innermost stable CBP orbit and $T_{\text {cov }}$ is the CoRoT observing duration. A modelling of their CoRoT light curves permitted the determination of the relative sizes and orbital inclinations of the stellar components. For some of these light curves, additional radial velocity observations were also obtained for a better determination of absolute and relative masses and sizes. Of several planet-like transit features detected by the algorithm, none survived on closer scrutiny. As a result, only coplanar CBPs of Saturn to Jupiter sizes, with periods between $P_{\text {min }}$ and $\approx T_{\text {cov }}$ could be excluded on these few binaries.

The active phase of the CoRoT mission finished in Nov. 2012, and the full data set became available a few months later. The availability of the full CoRoT mission data, the knowledge from Kepler that transiting CBPs exist and may be detectable, and the development of an improved detection algorithm, presented in Sect. 4, provided sufficient motivation for the renewed CBP search in CoRoT Data that is reported here.

In this paper we present a method for quasi-periodic transit detection, including the necessary selection and preparation of the binary light curves, and the results of this search for circumbinary planets in CoRoT Data. In Sect. 2 we outline several issues affecting CBP transit searches, with an overview of CBP detection algorithms. In Sect. 3 we give a brief description of the binary sample. In Sect. 4 we detail the method of the search and the code. Test results on artificial and real light curves, as well as on already known CBPs, are shown in Sect. 5. We present our results in Sect. 6 and discuss the planet candidates that were found. In Sect. 7 we interpret the search results and derive upper limits for the probabilities that detectable circumbinary planetary systems exist within the analysed sample of binaries. Final conclusions are given in Sect. 8.

\section{Issues on searches for transits of circumbinary planets}

The detection of transiting CBPs is very different from that of planets orbiting single stars. In the case of a CBP, the configurations when transits occur depend on the orbital phases of both the binary and the planet. Therefore, transits only occur quasi-periodically within a transit window that is recurrent with the period of the planet. Furthermore, the shapes of transit light curve can be very complex (e.g. Deeg et al. 1998), depending on the relative velocities of the three bodies, and are further complicated by planet transits happening during mutual binary transits. Indeed, Kepler $16 \mathrm{~b}$ and most of the first transiting CBPs were found from visual inspections, and only some more recent and more challenging discoveries (e.g. Kepler-413b, Kostov et al. 2014) were found with search algorithms. Nevertheless, the complexity of these curves provides a secure diagnostics to assure the planetary nature of such a detection, freeing transiting CBP detections from the worries about false alarms that are notorious in single star transit detections (e.g. Brown 2003; Almenara et al. 2009).

A first step in a CBP transit search is the removal of the binary eclipses. For this step, the binary eclipses may be modelled either with a physical model of the binary, which involves the fitting of physical binary parameters, or with a purely phenomenological description of the binary brightness variations, which may be derived from a light curve that is phased with the period of the binary. This second method is that used in this work. In either case, the removal of the binary might be affected by period changes in the binary orbit because of both evolutionary and dynamical effects that make it more difficult to fully remove the eclipse light curve. Non-eclipsing third components with stellar masses may also cause significant period variations. In the case of EB light curves in Kepler data, in some cases such variations were of the order of percents of the period itself (Borkovits et al. 2015). In the case of the CBP search in CoRoT light curves, however, we expect period variations to be negligible. On the one hand, the length of the light curves is limited to $\approx 150$ days, a time span over which period variations manifest themselves much less. On the other hand, we are interested in detecting objects with planetary masses, and such small masses do not cause period variations with relevant effects onto the binary light curve removal. In the known transiting CBPs from Kepler, the variations in EB periods over time spans of 150 days were all below one minute. Furthermore, any strong period variation that might 
be introduced by a stellar mass tertiary would at worst cause us to miss a planet detection in that particular system.

Besides the detection algorithm used in this work, which is outlined in more detail in Sect. 4, during the course of this work two other detection algorithms of interest for the detection of CBPs became published. The first detection algorithm is QATS (Carter \& Agol 2013), which searches for a most likely transit depth and duration that is subject to a quasi-periodic condition. That is, QATS is aimed at detecting events that have variations around a typical baseline condition, which is found in transits across single stars that suffer variations in periodicity, depth, and duration from the influence of other orbiting planets. QATS was used to detect several planets with strong transit timing variations, but its ability to detect CBPs, whose transits may have much more complicated deviations from a baseline-transit (e.g. Deeg et al. 1998), is still to be demonstrated ${ }^{2}$.

Another detection method was described in the context of the discovery of Kepler 64b (Kostov et al. 2013, given there as KIC 4862625), which was the first discovery of a CBP from the employment of a transit detection algorithm ${ }^{3}$. The method by Kostov et al. is based on the widely used BLS algorithm (Kovács et al. 2002), but is tuned to identify individual (instead of periodic) transit events as well as anti-transits, that is, brightening features with similar shapes. A statistical comparison of transit and anti-transit events using the method of Burke et al. (2006) leads to the identification of light curves in which true transit events may be present, thereby requiring further visual inspection. However, we do not expect this method to perform well on CoRoT light curves, since it assumes that any systematic flux variations are free of strong tendencies towards either transit or anti-transit-like events. In contrary to Kepler data, CoRoT Data do however suffer from frequent flux jumps that are mostly brightness increases (see Sect. 4.1.5) and neither are residuals from the correction of these jumps symmetric in transit or antitransit-like behaviour. We expect therefore that at least some modifications would be required for that method to work well on CoRoT Data.

\section{CoRoT binary sample}

For our CBP search, EBs were selected based on the CoRoT N3 automatic variable star classification output tables (Debosscher et al. 2009) ${ }^{4}$. In order to not miss any potential CBP host system, we selected all variables classified as EBs with nonzero probability (3188 targets). This means, in fact, that many of our targets are not binaries. In order to get a more reliable statistic of CBPs we inspected all these light curves visually and sorted out the non-eclipsing binary targets, leaving 1512 light curves in the list.

Additionally we merged a list of 1268 EBs identified with the DST transit detection tool (Cabrera et al. 2012), which was initially developed for the search of transiting planets in CoRoT Data. These binaries have generally low eclipse amplitudes of $<5 \%$ and have been published for some CoRoT

\footnotetext{
2 The only application of QATS to CBPs we are aware of is a search for a further planet in the two-planet CBP system Kepler 47, where QATS failed to detect the outer planet, with QATS termed by Orosz et al. (2012, in online supporting material) as "very sensitive to detrending errors for longer periods".

3 Simultaneously, its visual detection in Kepler light curves was reported as "PH1b" by Schwamb et al. (2013).

4 Tables available in

http://idoc-corotn2-public.ias.u-psud.fr
}

Table 1. Likely eclipsing binaries in CoRoT Data selected for the CBP search of this work.

\begin{tabular}{cc}
\hline \hline CoRoT-ID & Period \\
\hline 100552362 & 1.347850 \\
100588681 & 0.793206 \\
100619354 & 3.358470 \\
100624108 & 0.645742 \\
100657980 & 2.923230 \\
\hline
\end{tabular}

Notes. The period is the presumed binary orbital period from the procedure given in Sect. 4.1.4. The full table with 2290 targets is available at the CDS.

runs (IRa01, LRc01, SRc01, LRa01, LRa03 and SRa03) in Carpano et al. (2009), Cabrera et al. (2009), Erikson et al. (2012), Carone et al. (2012), Cavarroc et al. (2012). Since many targets were observed in more than one CoRoT run, the combination of these two lists results in 2780 light curves of 2290 individual binaries (see Table 1, published fully at the CDS).

We note that a list of EBs that is included in an upcoming paper describing the full set of CoRoT's transit detections (Deleuil et al., in prep.) has been derived independently from the list of EBs used in our work.

\section{Method for detection of CBP candidates in CoRoT Data}

Here we describe the method we developed for the search for transiting CBPs in the CoRoT EB light curves. The method is flexible enough to ingest light curves obtained by other telescopes (e.g. Kepler) as well with only slight modifications in the parameters during the light curve preparation.

First we present the code itself and how it works. Afterwards we show tests regarding the capability of the code and apply it to the known CBP Kepler-35b (Welsh et al. 2012).

\subsection{Light curve preparation}

In order to search for transit-like signals, ideally all flux variations should be removed except the transit-like features. These variations can be both of physical (eg. eclipses, stellar activity) and instrumental origin. In the case of CoRoT, sudden flux jumps are very common in the light curves and longer term trends may be present as well.

The main steps of the light curve preparation for each binary are as follows:

- selection of valid data points;

- binning to $512 \mathrm{~s}$;

- linear trend removal;

- binary period refinement and stellar eclipse removal;

- jump correction;

- additional light curve corrections.

\subsubsection{Data point selection}

We use the CoRoT N2 data release of 2013, starting from the provided fits files. Only data points with STATUS $=0$ are selected, which means we do not use any data flagged as being infected by cosmic rays or taken when the satellite crossed the South Atlantic Anomaly. 


\subsubsection{Data binning}

CoRoT Data contain two different time sampling modes, of $32 \mathrm{~s}$ and $512 \mathrm{~s}$ integration time, which could be alternated during the observations. In order to get consistent light curves with lower noises, we binned all $32 \mathrm{~s}$ observations to $512 \mathrm{~s}$.

\subsubsection{Linear trend removal}

Owing to several factors, such as the aging of the CCD cameras, the changing position of the sun relative to the satellite which is causing scattered light, and gradual recovery from cosmic-ray hits that occurred in previous pointings, CoRoT light curves may have slight linear gradients; these gradients amount to absolute flux variations of less than $2 \%$ across individual CoRoT runs ${ }^{5}$. To calculate more accurate averaged binary light curves it is beneficial to remove such gradients. Furthermore, in several light curves there are large instrumental jumps that cause unusable binned binary light curves (see Sect. 4.1.4). In these cases a linear fit to the whole light curve smooths the distribution of the data points.

\subsubsection{Binary period refinement and stellar eclipse removal}

Initial binary orbital periods (e.g. from the CoRoT N3 data product; Debosscher et al. 2009) may not be of sufficient accuracy for the removal of the stellar eclipses from the light curves. Therefore, before we subtract the eclipses, we refine the orbital periods. This is performed by trying different orbital periods around the catalogue value. For each test period we fold the light curve, divide it into bins, calculate the average in each bin, interpolate the binned points to the real data, subtract this from all points in the given bin, correct for instrumental jumps (see Sect. 4.1.5), and calculate the scatter of the whole processed light curve. The number of bins is $N_{\text {bin }}=2 \times \sqrt{N_{\text {data }}}$, where $N_{\text {data }}$ is the total number of data points and $N_{\text {bin }} \geq 200$, otherwise the phase curve is not sufficiently well sampled and parts of the eclipse signals may remain in the processed light curve. The accepted orbital period and final processed light curve are those where the scatter of the residuals is minimal. After this step the average of the processed light curve is 0 .

\subsubsection{Jump correction}

Jumps in the CoRoT light curves are well-known features (Mislis et al. 2010; Aigrain et al. 2009; Mazeh et al. 2009). These sudden flux changes range from several percent down to a few tenths of a percent. Usually these jumps occur as sudden flux increases that return gradually over days to weeks to previous values, although sudden resets to previous values have been observed as well. Also, in some cases, fluxes drop and return to their original value within a few hours, producing transit-like flux variations. Since in most cases these transit-like variations are present in one colour only (Mislis et al. 2010), they can be separated from real flux variations if the target lightcurve underwent CoRoT's chromatic processing leading to lightcurves in three colours, which was used on targets brighter than $\approx 14$ mag

\footnotetext{
5 Asensio-Torres (2016) found flux variations of $\$ 5 \%$ across the entire six-year CoRoT mission, based on zero-point magnitudes from absolute photometry. There was however a significant increase in noise across the mission; e.g. for an $R=14$ mag star, photometric errors over timescales of $2 \mathrm{~h}$ incremented by a factor of 2.2 .
}

(Ollivier et al. 2016). However, this separation is not possible for fainter targets that were observed only in monochromatic mode.

To find the position of the jumps, we step through all data points and calculate the median of the previous and next three days. At a jump, the difference of the two medians reaches a local maximum. These maxima are the borders of the light curve sections, which have to be shifted to the same level. The length of the section before and after the given point is selected so as not to confuse transits as jumps. The individual sections were fitted with a third order polynomial, which was then removed from the light curve sections.

\subsubsection{Additional light curve corrections}

So far we have not treated flux variations caused by stellar activity, which increases with shorter binary periods. Since our targets are mostly close binaries, it is crucial to deal with this effect. The method we apply is described by Cabrera et al. (2012) and uses a Savitzky-Golay filter. This method eliminates long-term light variations, such as spots on the rotating surface of stars, but keeps short transients, such as transit events, untouched. Variations within timescales of a few hours remain in the light curves, as these cannot be removed without degrading any transit signals as well.

After all these steps it is still possible to have some remnants of the eclipses in the processed light curves. This can be happen if the orbital period of the binary or the depth of the eclipses slightly changed during the observations. These remnants mimic periodic signals during the transit search and the real CBP transits may remain hidden. To avoid this, we fold the processed light curves with the binary orbital period, divide it into 200 bins and set all data points to zero in those bins, for which the following equation is true:

$\sigma_{\mathrm{bin}, i}>\overline{\sigma_{\mathrm{bin}}}+3 \times \sigma_{\sigma_{\mathrm{bin}, i}}$,

where $\sigma_{\text {bin, } i}$ is the standard deviation of data points in the $i$ th bin, $\overline{\sigma_{\text {bin }}}$ is the average of the standard deviation of the data points in the bins, while $\sigma_{\sigma_{\text {bin, } i}}$ is the standard deviation of the scatter in each of the bins $\left(\sigma_{\mathrm{bin}, i}\right)$.

\subsection{Search for quasi-periodic transits}

With the light curves treated as described, it is possible to search for transits in an automatic way. The algorithm is based on the derivation of the goodness of fit between transits of model planets and the processed data. The planet transits are modelled as simple strings of box-shaped transits that may shift to some extent against strict periodicity and which may have different durations and depths at each planetary orbit.

\subsubsection{Orbital period of model planets}

The innermost stable orbit of a planet around a binary system is $\sim 3$ times the orbital period of the binary (Dvorak et al. 1989). The actual value depends on the eccentricity of both the binary and the planet and the mutual inclination of the orbital planes (Holman \& Wiegert 1999). Therefore, we start the planet search with a conservative $P_{\mathrm{pl}}=2.6 P_{\text {bin }}$ and increment it up to half of $T_{\text {cov }}$, which is the length of the whole light curve. The steps are optimized to maintain some overlap between the searching phases incrementing from one period to the next, even at the end of the observations. A minimal planetary orbital period of 2.0 days was also imposed. Binaries with correspondingly short 
periods (e.g. $P_{\text {bin }}<0.77 d$ ) are usually very active contact binaries. Planet detection attempts in the $P_{\mathrm{pl}}<2$ day regime led to multitudes of peaks in the search statistic $S_{\text {total }}$ (see Sect. 4.2.4), which made it impossible to detect real planets in that regime.

\subsubsection{Transit parameters}

We model simple box-shaped transits, which have only two parameters, their depth and duration. Contrary to the case of a single star, in which all transits have the same depth and duration, in binary systems these parameters vary. The depth depends on the stellar component that is occulted, while the duration depends on the relative velocity of the transiting planet and stars.

In duration we model for 1, 2, 3, 4, 6, 10, and $16 \mathrm{~h}$-long transits. The transit depth may vary from $1.0 \times \sigma_{\mathrm{lc}}$ up to $0.04 \mathrm{mag}$, where $\sigma_{\mathrm{lc}}$ is the standard deviation of the processed light curve after the stellar eclipse removal, incrementing the depth in each step by a factor of $\sqrt{2}$. We also include a zero transit depth, since due to precession it may be possible that at some orbits no observable transits occur (e.g. Martin \& Triaud 2014, on the issue of the CBPs with sparse transits). These parameters are independent for each planetary orbit.

\subsubsection{Modelling of quasi-periodic events}

We fold the processed light curve using a model planet's period and epoch. Once for each planet orbit, a model transit is inserted whose duration and depth may vary between orbits and which we permit to shift in phase to allow for the quasi-periodic nature of the transits. The maximum phase shift depends on the ratio of the orbital period of the binary versus the period of the planet, with its limits given by

Shift $_{\max }= \pm \frac{1}{2 \pi} \times \arcsin \frac{P_{\text {bin }}^{2 / 3}}{P_{\mathrm{pl}}^{2 / 3}}$.

which is based on Kepler's third law with the planet of negligible mass, and Shift $t_{\max }$ is given in units of fractions of the test planet's orbital phase. Above equation leads to an overestimation, since it assumes that the entire mass of the central binary is concentrated in one component, while the true maximum elongation (of the components from the barycentre) is not the distance between the two stellar components, but the distance between the lower mass component and the barycentre. However, we do not take into account eccentric planetary or stellar orbits, which can increase the phase range of possible transits.

\subsubsection{Intermediate search statistic}

At each period step, we attempt to find the best fit against the box-shaped transit model. We evaluate all combinations of epoch, transit depth, phase shift, and transit duration, where the latter three parameters are able to vary between each planet orbit, and we save the best fit. The goodness of the fit for each set of parameters is given by the variance

$S_{\text {total }}=\sum_{i=1}^{N}\left(F_{\text {proc }, i}-F_{\text {test }, i}\right)^{2}$,

where $N$ is the number of data points in the light curve, $F_{\text {proc }, i}$ is the flux of the $i$ th point in the processed light curve, and $F_{\text {test }, i}$ is the flux of the $i$ th point in the modelled light curve.

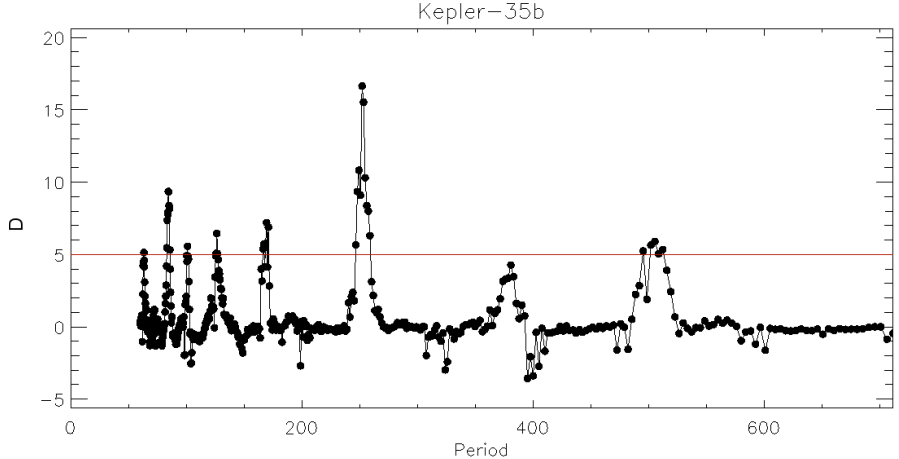

Fig. 1. Planet search result for Kepler-35b. The highest peak corresponds to twice the real orbital period, however, the true period at $\sim 125$ days is above the detection limit as well.

\subsubsection{Automatic detection}

If there is a quasi-periodic transit-like signal in the light curve, there will be corresponding peaks in an $S_{\text {total }}$ versus period diagram at the approximate period and at its harmonics. We detect peaks in a normalized version of $S_{\text {total }}$, using values of $S_{\text {total }}$ neighboring a given period to derive a local median and standard deviation of $S_{\text {total }}$,

$D_{p}=\frac{S_{\text {total }, p}-\operatorname{MEDIAN}\left(S_{\text {total, around } p}\right)}{\sigma_{S_{\text {total }, \text { around } p}}}$,

where $D_{p}$ is the detection statistic for the $p$ th period value

A peak in $S_{\text {total }}$ is considered significant if the difference between the peak's value and the median around the peak is $5 \sigma$ above the standard deviation of the points around the peak; that is, if $D>5.0$. This detection threshold was chosen as it leads to a number of detections that are feasible for a subsequent manual revision (as described in Sect. 6.2), yielding a signal in $\sim 18 \%$ of the binaries. Figure 1 shows an example that is discussed in more detail in Sect. 5.2.

Eighteen percent is much above the expected frequency of transiting CBPs. Less than $1 \%$ of the Kepler EBs have transiting planets and the expected number of detectable planets in the CoRoT light curves is even lower because of the lower photometric accuracy and shorter length of its observations. But since the transit signals are weak, we might miss planets if a higher threshold is used. The 506 light curves in which a signal was detected were then revised individually (see Sect. 6.2).

\section{Performance tests and detection probabilities}

\subsection{Test planets in real CoRoT Data}

In order to derive detection limits of the algorithm, we tested it on real CoRoT EB light curves with simulated transiting planets. Table 2 summarizes the results, in terms of detection probabilities (e.g. the ratio of detected and all test planets) versus the relative transit depth and the number of transits in a light curve, $N_{\mathrm{tr}}$. They were derived by the procedure described as follows.

Due to every light curve being individual, we used all the binaries of the sample described in Sect. 3, instead of selecting some subsets. In each EB light curve we inserted a test planet with a random orbital period distributed between $2.6 \times P_{\text {bin }}$ and half of the total data length $T_{\text {cov }}$. The transit depth $\Delta F$ was selected randomly between 0.5 and 5.0 times the scatter of the 
Table 2. Detection probability (the fraction of detected test planets) of the planet searching code on real CoRoT EB light curves.

\begin{tabular}{|l|l|l|l|l|l|l|l|l|l|l|}
\hline \hline \multirow{2}{*}{$N_{\text {tr }}$} & \multicolumn{70}{|c|}{$\Delta F$ : Transit depth in RMS units } & \multicolumn{1}{|c|}{ Total } \\
\cline { 2 - 10 } & $0.5-1.0$ & $1.0-1.5$ & $1.5-2.0$ & $2.0-2.5$ & $2.5-3.0$ & $3.0-3.5$ & $3.5-4.0$ & $4.0-4.5$ & $4.5-5.0$ & \\
\hline $2-3$ & $0.04(501)$ & $0.18(506)$ & $0.28(537)$ & $0.38(531)$ & $0.49(520)$ & $0.50(505)$ & $0.55(519)$ & $0.62(470)$ & $0.63(527)$ & 0.41 \\
$3-4$ & $0.04(257)$ & $0.29(253)$ & $0.46(251)$ & $0.63(239)$ & $0.62(231)$ & $0.71(240)$ & $0.71(243)$ & $0.73(251)$ & $0.81(238)$ & 0.55 \\
$4-5$ & $0.10(172)$ & $0.39(173)$ & $0.47(148)$ & $0.73(161)$ & $0.76(162)$ & $0.77(170)$ & $0.77(132)$ & $0.82(130)$ & $0.92(165)$ & 0.63 \\
$5-6$ & $0.16(322)$ & $0.48(310)$ & $0.66(285)$ & $0.78(258)$ & $0.85(298)$ & $0.88(321)$ & $0.90(301)$ & $0.92(308)$ & $0.94(328)$ & 0.73 \\
$6-7$ & $0.20(168)$ & $0.59(203)$ & $0.78(185)$ & $0.86(212)$ & $0.88(199)$ & $0.93(189)$ & $0.93(178)$ & $0.94(189)$ & $0.93(206)$ & 0.80 \\
$7-8$ & $0.27(348)$ & $0.64(332)$ & $0.79(321)$ & $0.89(318)$ & $0.94(313)$ & $0.95(352)$ & $0.96(347)$ & $0.96(297)$ & $0.96(324)$ & 0.81 \\
$8-9$ & $0.30(262)$ & $0.71(258)$ & $0.82(236)$ & $0.91(236)$ & $0.95(229)$ & $0.95(229)$ & $0.97(240)$ & $0.98(239)$ & $0.97(247)$ & 0.83 \\
$9-10$ & $0.38(194)$ & $0.71(173)$ & $0.89(187)$ & $0.95(173)$ & $0.95(177)$ & $0.96(184)$ & $0.99(183)$ & $0.96(177)$ & $0.99(163)$ & 0.86 \\
$10+$ & $0.55(730)$ & $0.88(726)$ & $0.94(719)$ & $0.96(684)$ & $0.98(666)$ & $0.98(767)$ & $0.98(778)$ & $0.99(746)$ & $0.99(728)$ & 0.92 \\
\hline
\end{tabular}

Notes. The total number of random tests performed for each box are in brackets. The accuracy of the probability values is 1 to $3 \%$. Colours indicate ranges of detection probability: red $<0.5$, yellow $0.5-0.8$, and green $>0.8$.

processed light curve, while the transit duration was chosen randomly between 1 and $16 \mathrm{~h}$ for each individual transit. The test was repeated several times, in some cases focusing on different regions of the $N_{\text {tr }}$ parameter to obtain similar numbers (of the order of a few hundreds) of tests in all of the cells of Table 2, resulting a total number of 25.975 tests. This means an average of nine different test planets were inserted in each CoRoT EB light curve. For each test planet we checked whether there is a significant detection peak at its orbital period, following the procedure of Sect. 4.2. The fraction of successful detections of all test planets in a given cell is presented in Table 2 .

With two transits in the light curve, the overall detection probability is $\sim 40 \%$, but for deep transits $\left(\Delta_{F}>3\right.$, where $\Delta_{F}=\delta F_{\mathrm{abs}} / \sigma_{\mathrm{lc}}$, with $\delta F_{\mathrm{abs}}$ being the absolute transit depth) it is above $80 \%$. For $N_{\text {tr }} \geq 3$ we are able to detect almost all test planets, except those with the shallowest transits with a depth of only $0.5 \times \sigma_{\text {lc }}$. These are the limits of the code.

As can be seen in Table 2, the code is able to find $>50 \%$ of the planets if the length of the observation $\left(T_{\operatorname{cov}}\right)$ is at least three times longer than the orbital period of the planet $\left(P_{\mathrm{pl}}\right)$; that is, if $N_{\mathrm{tr}} \geq 3$. The detection probability reaches $80 \%$ at $N_{\mathrm{tr}} \simeq$ $T_{\text {cov }} / P_{\mathrm{pl}}>6$ for the whole tested parameter space. If shallow transits with $\Delta_{F}<1.0$ are not taken into account, the $80 \%$ limit is reached at $N_{\mathrm{tr}}>5$ and the detection probability goes up to $96 \%$ for 10 or more observed transits.

Since the code is searching for quasi-periodic signals and due to its constraints on the model periods (Sect. 4.2.1), it should not be sensitive to mono-transits (transit-like events that appear only once in a given light curve). The code may however find such events, like the candidates discussed in Sect. 6.2, if there are other spurious signals, such as residuals from the jump correction or the eclipse removal.

\subsection{Kepler-35b}

As a test of our method using a different set of data, we attempted to identify the CBP Kepler-35b (Welsh et al. 2012) from its light curve provided by the third revision of the Kepler Eclipsing Binary Catalog (Matijevič et al. 2012). The published orbital period of the planet is $\sim 131.5$ days. The rms of the processed light curve after the preparation is $0.05 \%$, while the transit depth is $\sim 0.3 \%$, six times the rms. According to the results of the previous section this should be well detectable by our algorithm. The result in the form of the detection statistics $D$ versus the orbital period is shown in Fig. 1.

There are several peaks in that figure, which correspond to the orbital period of the planet and its harmonics. The peak near the published period of 131.5 days is at $\sim 125$ days with $D=$ 6.5. This difference is due to the relatively few transit events in the data with a large transit window and due to the design of the algorithm, which finds only approximate periods. The most significant peak at $P_{\mathrm{pl}} \sim 250$ days with $D \sim 17$ ) corresponds to $2 \times$ the true period. This feature is not real, but a characteristic of the code itself, and is a consequence of a high density of peaks in the $S$ statistics at shorter periods. Peaks in the $D$ values at these shorter periods become then less significant because the standard deviation in $S$ in the vicinity of such peaks is larger (see Eq. (4)). However, both the peak at the real period and its harmonic are above the detection limit of $D=5.0$, implying that the transit events in this light curve deserve closer scrutiny.

\section{Results}

\subsection{Residual noises in the processed light curves}

The first part of the planet search was the removal of the EB light curves and of variations in flux levels from cosmic rays or from instrumental effects. In an ideal case, such processed light curves contain only white noise and planetary transits, if they are present. However, it was impossible to remove all stellar activity variations as well as instrumental effects from the light curves, especially in short period systems, without destroying the transit signal.

Figure 2 shows the RMS of the light curves after the eclipse variation is removed, as a function of the $R$-band magnitude. Positive outliers are mainly due to stellar activity (e.g. shortterm fluctuations in the light curve), but incorrect jump removal may play a role as well. These light curves are not suitable for investigating the stellar activity of binaries because of the various corrections to optimize them for the transit search. However, variations on timescales of transit durations cannot be corrected as this would affect the transit signals.

\subsection{Planet candidates}

During the automatic search we found 506 binary light curves with detections above the $D=5$ limit (Red points in Fig. 2, 
P. Klagyivik et al.: Limits to the presence of transiting circumbinary planets in CoRoT Data

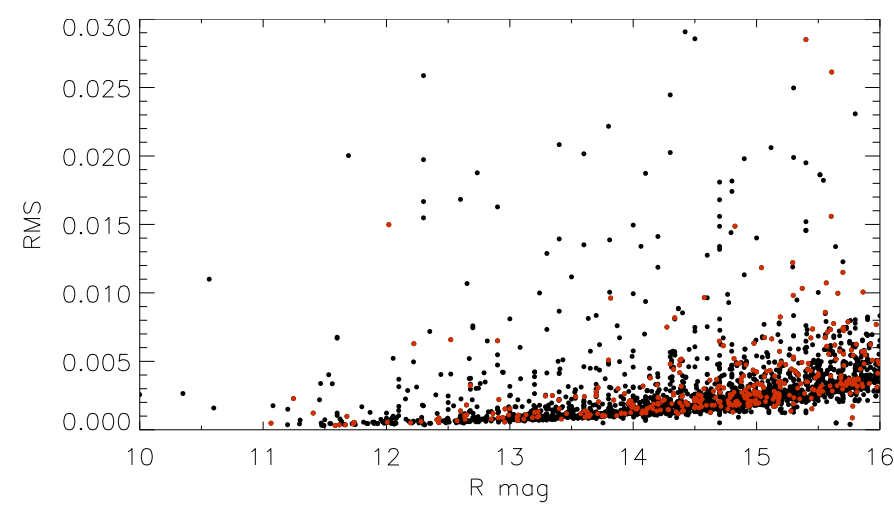

Fig. 2. RMS $\left(\sigma_{\mathrm{LC}}\right)$ of the processed light curves after the removal of stellar eclipses and instrumental effects. Red dots represent the 506 targets with detections above the threshold.

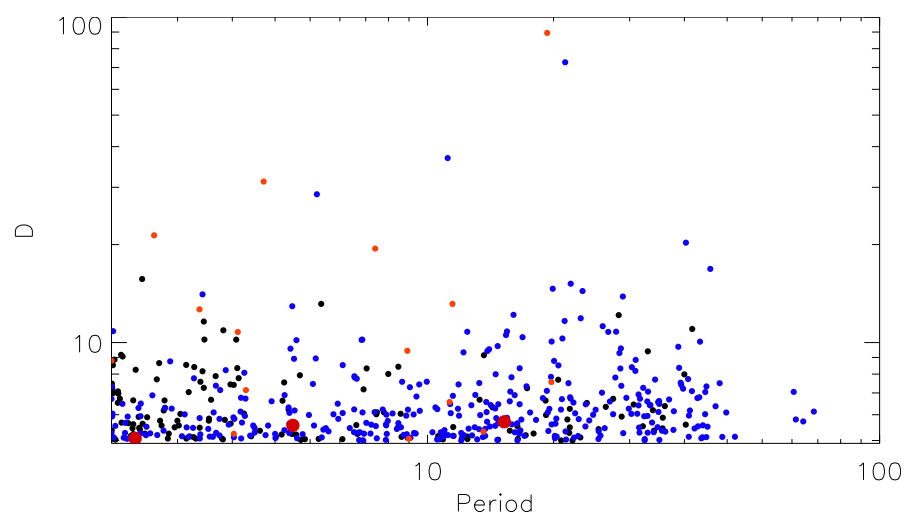

Fig. 3. Detection statistic $D$ vs. planet orbital period in days (on a $\log$ scale) in 506 light curves selected by the detection algorithm. A minimum search period of 2 days was imposed for the reasons given in Sect. 4.2.1. The colors of the points indicate classification from visual inspection; the red points indicate planet candidates; orange indicate background binaries; black represent stellar activity, and blue represent instrumental effects.

which shows the detection statistics as a function of the detected period). We inspected these light curves visually and separated them in five groups based on the origin of the detected signal. The groups are as follows: background binaries (orange points in Fig. 2, 21 systems), stellar variability (black, 143) and instrumental effects (blue, 339), and remaining planet candidates (red, 3).

The systems with the highest detection statistics belong to periodic variables, such as background EBs (cases with two EBs in a CoRoT aperture), spotted stars, or pulsating variables. In the long period regime, instrumental effects dominate, mainly because of smaller jumps still present in the light curves. These instrumental effect cannot be eliminated without modifying any real transit signals, as they are in the same timescale and amplitude range.

Several preliminary candidates of suspected planet transits were found, although all of them were potential mono-transit events. The lightcurves of the candidates observed in CoRoT's chromatic mode were checked in their three colors to recognize events with strong colour dependencies. Unfortunately all of these candidates were false alarms from instrumental effects. The candidates observed in monochromatic mode were checked individually, and only three of these candidates remained for further investigation. These final planet candidates are listed in Table 3. In Fig. 4 we present for each suspected planetary transit
Table 3. The three final planet candidates discussed individually in Sect. 6.2.

\begin{tabular}{lcc}
\hline \hline CoRoT-ID & $\begin{array}{c}\text { transit time } \\
\text { CHJD }\end{array}$ & $P_{\text {binary }}$ \\
\hline 310190466 & 3433.9 & 1.63353 \\
629951504 & 4222.9 & 0.26141 \\
634075176 & 4589.2 and 4591.9 & 5.02718 \\
\hline
\end{tabular}

Notes. The rightmost column contains the orbital period of the central binary.

the original light curve and the processed light curve after the stellar eclipse removal.

CoRoT-ID 310190466 is a relatively faint target with a brightness of $R=15.9$ magnitude. Despite the large rms (0.0062 magnitude) there is a clear transit-like event at CHJD 3433.9 (CHJD is the CoRoT heliocentric Julian date, given by CHJD $=$ HJD -2451545.0$)$. The depth of the suspected transit is $1.5 \%$, while the duration is $\sim 9 \mathrm{~h}$. The binary itself is an Algoltype, detached binary with an orbital period of 1.63353 days.

CoRoT-ID 629951504 is a short period contact binary with an orbital period of 0.26141 days. The suspected transit is at CHJD 4222.9. The depth is $0.5 \%$ and the duration is $\sim 7 \mathrm{~h}$. Since the transit shape could be very complex around a contact binary system, the small peak in the middle of the transit might be real, but is most probably only noise.

CoRoT-ID 634075176 is a detached binary. The orbital period is 5.02718 days. There are two transit-like events in the light curve close to each other at CHJD 4589.2 and 4591.9. The depths are both $0.4 \%$, while the durations are $\sim 3 \mathrm{~h}$ and $\sim 9 \mathrm{~h}$, respectively. The residual feature at 4588.3 is likely an instrumental jump. Folding the light curve of the binary, there is a shallow secondary eclipse at the orbital phase of 0.63 , with an amplitude of $\sim 0.003$, which implies stellar components of strongly different surface brightness. Therefore the transit candidates at $\mathrm{CHJD}=4589.2$ and 4591.9 are unlikely to be from a planet transiting both components, since their similar depths would require stellar components of approximately equal surface brightness.

The code is not optimized for finding single events. This means that mono-transits from longer period planets would only be found if there is some other transit-like event in the light curve (e.g. small jumps or eclipse residuals due to period change). Otherwise the mono-transits remain hidden. In order to find these transits we checked all light curves visually searching for single transit-like events, but we did not find any additional candidates.

Regarding the large number of detections that all turned out to be false alarms, which might leave doubts regarding the efficiency of the algorithm, we note that many of these false alarms have been caused by features that are characteristic of CoRoT Data. Similar situations may however also occur with data from the Kepler mission. When Boyajian et al. (2015) attempted an automatic search for further light curves with features similar to the unusual object KIC 8462852, they initially encountered over 1000 candidates. Their revision then led however to discarding of all of them, which in some cases was caused by instrumental effects. Similar to our search, their algorithm was also relatively broad in the requirements that led to a detection, which was tuned to avoid the oversight of potential discoveries. As long as the revision of the detections that are encountered by such algorithms is manageable, be it manually or by specific algorithms, this is a correct approach. 

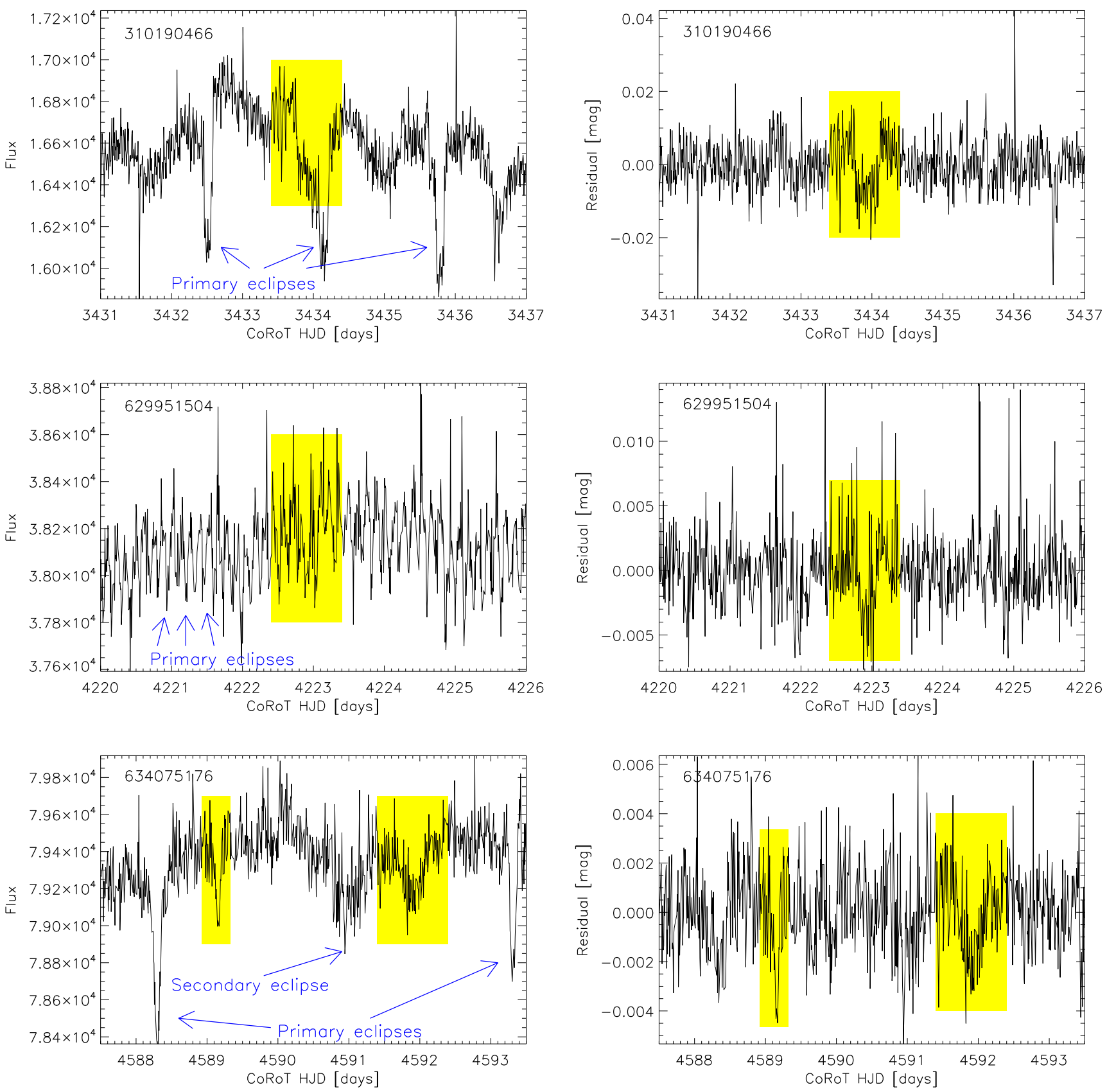

Fig. 4. Light curves around potential transits (yellow background) of the three planet candidates. The original light curves are on the left side, with some stellar eclipses indicated, while the processed light curves after the stellar eclipse removal are on the right side. Time is in CoRoT heliocentric Julian date, which is HJD - 2451545.

\section{Limits to the occurence rate of circumbinary planets}

There are multiple works in the literature that establish abundances of some planet populations from extrapolation of known abundances of somewhat different populations. For example, works about eta-Earth (abundance of Earth-like planets) extrapolate from known abundances of larger or hotter planets; e.g. Petigura et al. (2013), Foreman-Mackey et al. (2014), Burke et al. (2015). In this work, however, on one hand, we are confronted with non-detections, and on the other hand, the sample of known CBPs is very scarce and permits only very limited comparisons. We therefore attempt here to derive upper abundance limits for given CBP populations based on the absence of detections in our sample.

We calculate the upper limit of the potential abundance of a given planet in our EB sample by using the binomial distribution. The probability $P$ of getting exactly $k$ successes in $n$ trials is given by the probability mass function,

$P=\left(\begin{array}{l}n \\ k\end{array}\right) p^{k}(1-p)^{n-k}$

where $p$ is the probability for the success of each individual trial. In our case $p$ is the unknown probability that a transiting CBP exists around an EB with a given set of parameters. In the case 
of a non-detection, we have $k=0$ and then obtain

$P(k=0)=\left(\begin{array}{l}n \\ 0\end{array}\right) p^{0}(1-p)^{n}$

which can be solved for $p$,

$p=1-P(k=0)^{1 / n}$.

Since we are interested in approximate upper limits to the CBP planet abundance from non-detections, we set $P(k=0)$ to 0.5 . This means that if the true fraction of planets with a given parameter set is $p$, than there is $50 \%$ chance that such planets would not have been detected in the sample. The true value of $p$ from a non-detection remains however unknown and might be much lower, so we can only determine reasonable upper limits $p_{\max }$, given by

$p_{\max } \leq 1-P(k=0)^{1 / n}=1-0.5^{1 / n}$.

For more conservative values of $p_{\max }$, the probability of getting no detection in $n$ trials, $P(k=0)$ should be set to lower values.

The probability that a light curve in our EB sample is suitable to contain the potential detection of a CBP of a given period depends on the length of the light curve and the period of the EB. In turn, the number $N_{\mathrm{s}}$ of binary light curves in the CoRoT sample that are suitable for the detection of a planet of a given period depends on that period. The lengths of the light curves vary from 23 days (SRa01 field) to 152 days (LRc01 field), and only light curves that are longer than twice the orbital period of a potential planet were considered suitable in order to assure that $N_{\text {tr }} \geq 2$. Furthermore, for a given planet period, only light curves of binaries with periods shorter than $1 / 3.5 \times$ the planet period are suitable, to consider only stable orbits. The combination of these two requirements led to the values $N_{\mathrm{s}}$ that are shown in Table 4, for different sets of binary periods.

Moreover, the detection probability of our code, $p_{\text {det }}$, is not 1.0 , therefore an effective number $N_{\text {eff }}$ of suitable systems for the detection of a planet with given absolute transit depth $\delta F_{\text {abs }}$ and period $P_{\mathrm{pl}}$ (or number of transits in the light curve, $N_{\mathrm{tr}}$ ) is calculated as

$N_{\mathrm{eff}}=\sum_{N_{\mathrm{s}}} p_{\text {det }}\left(\Delta F, N_{\mathrm{tr}}\right)$,

where $p_{\text {det }}\left(\Delta F, N_{\text {tr }}\right)$ are the values from Table 2,in which the relative transit depth $\Delta F$ and transit counts $N_{\text {tr }}$ were calculated individually for each of the light curves within $N_{\mathrm{s}}$. For light curves for which a given test planet results in relative transit depths of $\Delta F<0.5, p_{\text {det }}$ was set to 0 . Inserting $N_{\text {eff }}$ as $n$ into Eq. (7), we are able to obtain upper limits on the probability of existence of transiting planets with $\left(P_{\mathrm{pl}}\right)$ and absolute transit depths $\delta F_{\text {abs }}$ in the CoRoT EB sample.

In Table 4, we give the upper limits $p_{\max }$ for the abundances of transiting Jupiter-, Saturn-, and Neptune-sized CBPs, assuming corresponding transit depths of $\delta F_{\text {abs }}=0.5 \%, 0.35 \%$ and $0.05 \%$. These values for $\delta F_{\text {abs }}$ correspond to planets with radii of $1 R_{\mathrm{Jup}}, 0.8 R_{\mathrm{Jup}}$, and $0.3 R_{\mathrm{Jup}}$, respectively, transiting one component of a binary of two solar-like stars (with radii of $1 R_{\odot}$ ). For each of these planets, the table gives $p_{\max }$ for planetary orbital periods $P_{\mathrm{pl}}$ up to 10,25, and 50 days around binaries with orbital periods $P_{\text {bin }}$ less than $1.0,2.5,5.0$, and 10.0 days. The inner stability limit of the planets was set to 3.5 times the orbital period of the binary. This means that for example for $P_{\text {bin }}<1.0$ day and $P_{\mathrm{pl}}<10.0$ days, the upper abundance limit $p_{\max }$ corresponds to planets with periods between 3.5 and 10.0 days. Since we calculate upper limits, $N_{\text {tr }}$ was calculated using the longest planetary orbital period in the given period interval, which corresponds to the lowest $p_{\text {det }}$ values and consequently to the highest limits. For cases with $N_{\text {eff }}<5$, the CoRoT Data do not place useful constraints on the planet abundances $p_{\max }$, they are indicated by red values in Table 4 . The smallest possible planet on a 10 day period with a potential to be found in any of the CoRoT binaries has a size of $\sim 2.5 R_{\text {Earth }}$.

In the case of so-called misaligned planets, that is, those with a relevant angle between binary and planetary orbital planes, it is possible that transits occur only in a small fraction of planetary orbits because of the precession of the orbital plane of the planet. The time spent in transitability, that is, when transits may occur owing to correct inclinations, depends on the inclination of the binary against the line of sight and on the mutual inclination $\Delta I$ between the planet and binary orbital planes. A detailed description of the geometry and transitability of misaligned CBPs is given by Martin \& Triaud (2015). However, if the misalignment of a planet is low enough, there is a transit in every planetary orbit (100\% transitability), regardless of the orbital phase of the binary. From basic geometric considerations and assuming an edge-on $\left(i=90^{\circ}\right)$ binary with components of 1 solar radii, we can therefore derive that the values in Table 4 are valid for mutual inclinations within $\Delta I= \pm 4.7^{\circ}, \pm 2.5^{\circ}$, and $\pm 1.6^{\circ}$ for orbital periods of 10,25 , and 50 days, respectively. We note that the largest mutual inclination among the known transiting CBPs is $4.1^{\circ}$ on Kepler $413 \mathrm{~b}$ (Kostov et al. 2014). This planet has a period of 66 days around two smaller stars (of 0.78 and $0.48 R_{\text {sol }}$ ) and hence displays year-long stretches without transits, on an 11year precession period. The majority of known CBPs have however small mutual inclinations; for example Kostov et al. (2014) quote an average of $0.3^{\circ}$ for the other CBPs known at that time, from which transit events at most of their planet orbits can be expected.

The fraction of single stars with hot Jupiters $(P<10$ days $)$ is $1.2 \% \pm 0.38 \%$ from the Doppler sample of Wright et al. (2012) and $0.6 \% \pm 0.1 \%$ from the Kepler photometric sample (Wang et al. 2015b). The difference is mainly due to different stellar types. In our binary sample the abundance limit for CBPs of similar size and $P<10$ days is $p_{\max }=0.21 \%$ for $P_{\text {bin }}<1.0$ days and $p_{\max }=0.09 \%$ for $P_{\text {bin }}<2.5$ days. These results suggest that short-period gas giants in binary systems are relatively rare or that most such planets have highly misaligned orbits.

\section{Conclusions}

A catalogue of eclipsing binaries in CoRoT Data was collected from the automatic classification results published in CoRoT N3 data and from EBs that were identified with an algorithm used for the search for transiting planets around single stars. A total of 2780 EB systems were selected on which a search for circumbinary planets was performed.

A method for the detection of transiting circumbinary planets in EB light curves was developed. Performance tests show that we are able to detect $>50 \%$ of the test planets if the transit depth is $>3 \times$ the rms noise of the processed light curve, after the removal of stellar eclipses and instrumental jumps, even if there are only two transits in the light curve. The detection probability is $>80 \%$ if there are at least five transits. A test of this method was performed on a difficult detection in data from the Kepler mission, namely the circumbinary planet Kepler-35b discovered 
Table 4. Upper limits for the abundance of transiting CBPs in dependence of binary and planetary periods.

\begin{tabular}{|c|c|c|c|c|c|c|c|c|c|c|c|c|}
\hline \multirow[b]{2}{*}{$\begin{array}{c}P_{\text {bin }} \\
\text { (days) }\end{array}$} & \multicolumn{4}{|c|}{$P_{\mathrm{pl}}<10$ days } & \multicolumn{4}{|c|}{$P_{\mathrm{pl}}<25$ days } & \multicolumn{4}{|c|}{$P_{\mathrm{pl}}<50$ days } \\
\hline & $\begin{array}{l}p_{\max } \\
(\%)\end{array}$ & $N_{\mathrm{s}}$ & $N_{\text {eff }}$ & $\begin{array}{c}P_{\mathrm{pl}} \text { range } \\
(\text { days })\end{array}$ & $\begin{array}{l}p_{\max } \\
(\%)\end{array}$ & $N_{\mathrm{s}}$ & $N_{\text {eff }}$ & $\begin{array}{c}P_{\mathrm{pl}} \text { range } \\
\text { (days) }\end{array}$ & $\begin{array}{l}p_{\max } \\
(\%)\end{array}$ & $N_{\mathrm{s}}$ & $N_{\text {eff }}$ & $\begin{array}{c}P_{\mathrm{pl}} \text { range } \\
\text { (days) }\end{array}$ \\
\hline \multicolumn{13}{|c|}{ Jupiter-sized planet $\left(\delta F_{\text {abs }}=0.5 \%\right)$} \\
\hline$<1.0$ & 0.21 & 534 & 322.83 & $3.5-10.0$ & 0.44 & 350 & 157.550 & $3.5-25.0$ & 2.06 & 95 & 33.36 & $3.5-50.0$ \\
\hline$<2.5$ & 0.09 & 1151 & 748.60 & $8.75-10.0$ & 0.17 & 774 & 407.911 & $8.75-25.0$ & 0.56 & 318 & 123.24 & $8.75-50.0$ \\
\hline$<5.0$ & - & - & - & - & 0.11 & 1131 & 635.111 & $17.5-25.0$ & 0.33 & 514 & 210.18 & $17.5-50.0$ \\
\hline$<10.0$ & - & - & - & - & - & - & - & - & 0.25 & 663 & 277.51 & $35.0-50.0$ \\
\hline \multicolumn{13}{|c|}{ Saturn-sized planet $\left(\delta F_{\mathrm{abs}}=0.35 \%\right)$} \\
\hline$<1.0$ & 0.46 & 534 & 149.52 & $3.5-10.0$ & 1.33 & 350 & 51.58 & $3.5-25.0$ & 6.27 & 95 & 10.71 & $3.5-50.0$ \\
\hline$<2.5$ & 0.17 & 1151 & 407.35 & $8.75-10.0$ & 0.41 & 774 & 170.14 & $8.75-25.0$ & 1.38 & 318 & 49.76 & $8.75-50.0$ \\
\hline$<5.0$ & - & - & - & - & 0.24 & 1131 & 289.01 & $17.5-25.0$ & 0.75 & 514 & 92.42 & $17.5-50.0$ \\
\hline$<10.0$ & - & - & - & - & - & - & - & - & 0.56 & 663 & 122.54 & $35.0-50.0$ \\
\hline \multicolumn{13}{|c|}{ Neptune-sized planet $\left(\delta F_{\mathrm{abs}}=0.05 \%\right)$} \\
\hline$<1.0$ & 7.75 & 534 & 8.59 & $3.5-10.0$ & 24.3 & 350 & 2.49 & $3.5-25.0$ & 62.8 & 95 & 0.70 & $3.5-50.0$ \\
\hline$<2.5$ & 1.50 & 1151 & 45.91 & $8.75-10.0$ & 4.87 & 774 & 13.88 & $8.75-25.0$ & 16.3 & 318 & 3.89 & $8.75-50.0$ \\
\hline$<5.0$ & - & - & - & - & 2.57 & 1131 & 26.61 & $17.5-25.0$ & 8.88 & 514 & 7.45 & $17.5-50.0$ \\
\hline$<10.0$ & - & - & - & - & - & - & - & - & 6.73 & 663 & 9.95 & $35.0-50.0$ \\
\hline
\end{tabular}

Notes. Red numbers indicate unreliable values given that $N_{\text {eff }}<5$.

by Welsh et al. (2012). The code found this planet's shallow transits, with a detection above the threshold for planet candidates.

We did not find any planet candidates with transits from two or more orbits in the data from CoRoT. However, we found three marginal candidates with transit events that would have arisen during one orbital revolution alone. One of these, CoRoT-ID 634075176, with an apparent double transit, is unlikely to be a planet because the depth of the transits is incompatible with the surface-brightness ratio of the stellar components.

Upper limits for the abundance of Jupiter-, Saturn-, and Neptune-sized planets in co-planar orbits for up to 10, 25, and 50 days orbital periods were derived. Our results imply that shortperiod giant planets $(P<10$ days $)$ are less frequent around binary systems than around single stars. A less likely option, that short period giant CBPs are preferentially on highly misaligned orbits cannot be excluded however. These results are also valid for slightly misaligned planets as long as such planets would generate transits at each planetary orbit.

Because of the limited temporal coverage of the CoRoT pointings, the analysed sample is mainly suitable for the search for short-periodic CBPs. The CBP with the shortest known orbital period is Kepler $47 \mathrm{~b}$, with a period of 49.5 days, which orbits a binary with a 7.45 day period. CoRoT would have been reasonably sensitive to a CBP with such an orbital period, but much less (or not at all) sensitive to any of the other, longer periodic CBPs found by Kepler. In Kepler data, it has also been noted that most of the CBPs orbit near the inner stability limit, while none of the CBPs orbit binaries with periods of less than seven days. Given that detection probabilities in Kepler data are also higher for the detection of short-periodic CBPs than for longer
CBPs, the non-detection of CBPs in CoRoT Data supports the pronounced absence of short-periodic CBPs. Our results therefore support the claim by Martin et al. (2015) and Hamers et al. (2016) that a third stellar-mass companion is needed for the formation of short-periodic binaries, with the third companion preventing also the presence of planets near the inner stability limit.

Acknowledgements. We thank the referee, Jon Jenkins, for comments that led to significant improvement in the presentation of this work. The authors wish to thank the entire CoRoT team, who made the generation of the data used in this work possible. The CoRoT space mission was developed and operated by CNES, with contributions from Austria, Belgium, Brazil, ESA, Germany, and Spain. CoRoT Data are available to the public from the CoRoT archive at http: //idoc-corot.ias.u-psud.fr. P.K. and H.D. acknowledge support by grants AYA2012-39346-C02-02 and ESP2015-65712-C5-4-R of the Spanish Secretary of State for R\&D\&i (MINECO). This project has been supported by the Hungarian National Research, Development and Innovation Office - NKFIH K-115709 and by the Hungarian OTKA Grant K113117. We would like to thank Antonio Dorta for his help in the supercomputations. This paper made use of the IAC Supercomputing facility HTCondor (http://research.cs.wisc.edu/ htcondor/), partly financed by the Ministry of Economy and Competitiveness with FEDER funds, code IACA13-3E-2493.

\section{References}

Aigrain, S., Pont, F., Fressin, F., et al. 2009, A\&A, 506, 425 Almenara, J. M. 2010, Ph.D. Thesis, Universidad de La Laguna, Spain Almenara, J. M., Deeg, H. J., Aigrain, S., et al. 2009, A\&A, 506, 337 Asensio-Torres, R. 2016, ArXiv e-prints [arXiv: 1609.02436]

Baran, A. S., Zola, S., Blokesz, A., Østensen, R. H., \& Silvotti, R. 2015, A\&A, 577, A146

Beuermann, K., Hessman, F. V., Dreizler, S., et al. 2010, A\&A, 521, L60

Borkovits, T., Rappaport, S., Hajdu, T., \& Sztakovics, J. 2015, MNRAS, 448, 946 
P. Klagyivik et al.: Limits to the presence of transiting circumbinary planets in CoRoT Data

Borucki, W. J., \& Summers, A. L. 1984, Icarus, 58, 121

Boyajian, T. S., LaCourse, D. M., Rappaport, S. A., et al. 2015, MNRAS, 457, 3988

Brown, T. M. 2003, ApJ, 593, L125

Burke, C. J., Gaudi, B. S., DePoy, D. L., \& Pogge, R. W. 2006, AJ, 132, 210

Burke, C. J., Christiansen, J. L., Mullally, F., et al. 2015, ApJ, 809, 8

Cabrera, J., Fridlund, M., Ollivier, M., et al. 2009, A\&A, 506, 501

Cabrera, J., Csizmadia, S., Erikson, A., Rauer, H., \& Kirste, S. 2012, A\&A, 548 A44

Carone, L., Gandolfi, D., Cabrera, J., et al. 2012, A\&A, 538, A112

Carpano, S., Cabrera, J., Alonso, R., et al. 2009, A\&A, 506, 491

Carter, J. A., \& Agol, E. 2013, ApJ, 765, 132

Cavarroc, C., Moutou, C., Gandolfi, D., et al. 2012, Ap\&SS, 337, 511

Debosscher, J., Sarro, L. M., López, M., et al. 2009, A\&A, 506, 519

Deeg, H. J., Doyle, L. R., Kozhevnikov, V. P., et al. 1998, A\&A, 338, 479

Deeg, H. J., Moutou, C., Erikson, A., et al. 2010, Nature, 464, 384

Doyle, L. R., Dunham, E. T., Deeg, H.-J., Blue, J. E., \& Jenkins, J. M. 1996, J. Geophys. Res., 101, 14823

Doyle, L. R., Deeg, H. J., Kozhevnikov, V. P., et al. 2000, ApJ, 535, 338

Doyle, L. R., Carter, J. A., Fabrycky, D. C., et al. 2011, Science, 333, 1602

Dvorak, R., Froeschle, C., \& Froeschle, C. 1989, A\&A, 226, 335

Erikson, A., Santerne, A., Renner, S., et al. 2012, A\&A, 539, A14

Foreman-Mackey, D., Hogg, D. W., \& Morton, T. D. 2014, ApJ, 795, 64

Gilliland, R. L., Jenkins, J. M., Borucki, W. J., et al. 2010, ApJ, 713, L160

Hale, A., \& Doyle, L. R. 1994, Ap\&SS, 212, 335

Hamers, A. S., Perets, H. B., \& Portegies Zwart, S. F. 2016, MNRAS, 455, 3180

Holman, M. J., \& Wiegert, P. A. 1999, AJ, 117, 621

Jenkins, J. M., Doyle, L. R., \& Cullers, D. K. 1996, Icarus, 119, 244
Jenkins, J. M., Caldwell, D. A., Chandrasekaran, H., et al. 2010, ApJ, 713, L120

Kostov, V. B., McCullough, P. R., Hinse, T. C., et al. 2013, ApJ, 770, 52

Kostov, V. B., McCullough, P. R., Carter, J. A., et al. 2014, ApJ, 784, 14

Kovács, G., Zucker, S., \& Mazeh, T. 2002, A\&A, 391, 369

Kuzuhara, M., Tamura, M., Ishii, M., et al. 2011, AJ, 141, 119

Martin, D. V., \& Triaud, A. H. M. J. 2014, A\&A, 570, A91

Martin, D. V., \& Triaud, A. H. M. J. 2015, MNRAS, 449, 781

Martin, D. V., Mazeh, T., \& Fabrycky, D. C. 2015, MNRAS, 453, 3554

Matijevič, G., Prša, A., Orosz, J. A., et al. 2012, AJ, 143, 123

Mazeh, T., Guterman, P., Aigrain, S., et al. 2009, A\&A, 506, 431

Mislis, D., Schmitt, J. H. M. M., Carone, L., Guenther, E. W., \& Pätzold, M. 2010, A\&A, 522, A86

Ollivier, M., Deru, A., Chaintreuil, S., et al. 2016, in The CoRoT Legacy Book: The adventure of the ultra high precision photometry from spaces (EDP Sciences), 41

Orosz, J. A., Welsh, W. F., Carter, J. A., et al. 2012, Science, 337, 1511

Petigura, E. A., Howard, A. W., \& Marcy, G. W. 2013, Proc. Nat. Acad. Sci., 110, 19273

Potter, S. B., Romero-Colmenero, E., Ramsay, G., et al. 2011, MNRAS, 416, 2202

Schneider, J. 1994, Planet. Space Sci., 42, 539

Schneider, J., \& Chevreton, M. 1990, A\&A, 232, 251

Schneider, J., \& Doyle, L. R. 1995, Earth Moon Planets, 71, 153

Schwamb, M. E., Orosz, J. A., Carter, J. A., et al. 2013, ApJ, 768, 127

Wang, J., Fischer, D. A., Barclay, T., et al. 2015a, ApJ, 815, 127

Wang, J., Fischer, D. A., Horch, E. P., \& Huang, X. 2015b, ApJ, 799, 229

Welsh, W. F., Orosz, J. A., Carter, J. A., et al. 2012, Nature, 481, 475

Wright, J. T., Marcy, G. W., Howard, A. W., et al. 2012, ApJ, 753, 160 